CORRIGENDUM

doi:10.1038/nature14334

\title{
Corrigendum: Human gut Bacteroidetes can utilize yeast mannan through a selfish mechanism
}

Fiona Cuskin, Elisabeth C. Lowe, Max J. Temple, Yanping Zhu, Elizabeth A. Cameron, Nicholas A. Pudlo, Nathan T. Porter, Karthik Urs, Andrew J. Thompson, Alan Cartmell, Artur Rogowski, Brian S. Hamilton, Rui Chen, Thomas J. Tolbert, Kathleen Piens, Debby Bracke, Wouter Vervecken, Zalihe Hakki, Gaetano Speciale, Jose L. Munōz-Munōz, Andrew Day, Maria J. Peña, Richard McLean, Michael D. Suits, Alisdair B. Boraston, Todd Atherly, Cherie J. Ziemer, Spencer J. Williams, Gideon J. Davies, D. Wade Abbott, Eric C. Martens

\& Harry J. Gilbert

Nature 517, 165-169 (2015); doi:10.1038/nature13995

In this Article focusing on the selfish metabolism of yeast mannan by Bacteroidetes, we also described a polysaccharide utilization locus (PUL) responsible for the degradation of high mannose mammalian $\mathrm{N}$-glycan (HMNG) but omitted to cite two relevant papers ${ }^{1,2}$, for which we apologise. Both studies describe a model for the degradation of complex biantennary $N$-glycans by Bacteroidetes in which the degradative enzymes are encoded by PULs. These studies ${ }^{1,2}$ provide examples of how PULs can orchestrate $N$-glycan metabolism in addition to the HMNG PUL we describe in this Article. In all three papers it is proposed that $N$-glycan depolymerization occurs primarily in the periplasm.

1. Renzi, F. et al. The N-glycan glycoprotein deglycosylation complex (Gpd) from Capnocytophaga canimorsus deglycosylates human IgG. PLoS Pathog. 7, e1002118 (2011).

2. Nihira, T. et al. Discovery of $\beta$-1,4-D-mannosyl- $N$-acetyl-D-glucosamine

phosphorylase involved in the metabolism of $\mathrm{N}$-glycans. J. Biol. Chem. 288, 27366-27374 (2013). 